\title{
A Feasibility Study of SOA-enabled Networked Rock Bolts
}

\author{
Jens Eliasson, Pablo Puñal Pereira, \\ Henrik Mäkitaavola, Jerker Delsing, Joakim Nilsson \\ Dept. of Computer science, Electrical and Space Engineering \\ Luleå University of Technology \\ Luleå, Sweden \\ Email: jens.eliasson@1tu.se
}

\author{
Joakim Gebart \\ Eistec AB \\ Luleå, Sweden \\ Email: joakim.gebart@eistec.se
}

\begin{abstract}
The use of rock bolts in the mining industry is a widely used approach for increasing mine stability. However, when compared to the automation industry, where the use of sensors and real-time monitoring of processes have evolved rapidly, the use rock bolts have not changed a lot during the last 100 years. What is missing are technologies for keeping installed rock bolts under real-time and online monitoring. One problem is that rock bolts can become damaged by seismic activities or movements within the rock, and thus lose their load bearing capacity. If that happens, the outer shell of a tunnel's walls or ceiling can collapse, with disaster as a result. Therefore, there is a clear need for online and real-time monitoring solutions for strain and thereby stress, as well as seismic activity.

In this paper, the current state of art in research around intelligent rock bolts is presented. An intelligent rock bolt is the combination of a traditional rock bolt with an Internet of Things device, i.e. a rock bolt with embedded sensors, actuators, processing capabilities and wireless communication. In the proposed architecture, every rock bolt has its own IPv6 address and can establish a wireless mesh network in an ad-hoc manner. By measuring strain and seismic activity and exposing the sensors in the form of services, large gains in terms of safety and efficiently can be achieved. A number of mining related activities such as stress on the rock bolt can be detected, falling rocks and the presence of mobile machinery can be observed. Since the network is based on standard communication protocols such as IPv6, it is vital to add security mechanisms to prevent eavesdropping and tampering of data traffic.

By utilizing the real-time monitoring capabilities of a network of Internet-connected intelligent rock bolt, it is possible to drastically improve monitoring of mining activities and thereby providing workers with a safer working environment.
\end{abstract}

\section{INTRODUCTION}

The importance of mining for the European Union (EU) has been well documented [1] and its role in the world economy as well [2]. Mining produces raw materials for use in various industries such as car manufacturing, ship building, construction of houses and infrastructure, etc., to mention a few [3]. The mining industry produces materials and products in the range of 84.2 billion euros per year, and employs more than 615 thousand workers within the European Union [1].

During the mining process, which is going deeper than ever before, long tunnels deep within a mountain are created by a combination of drilling, followed by the use of explosives in order to break solid rock in smaller pieces that can be transported by for example haulers, elevators, conveyer belts, or trucks from the mine site to a process plant at the surface. At the process plant, grinders are used to crush large pieces of rock into small enough pieces that can be used in the process of creating iron ore and iron pellets. During this process, large voids are created down in the mine, which can cause instabilities and even lead to smaller earthquakes. In some cases, micro seismic activity can even lead to collapse in tunnels, causing injuries and even death to workers as well as substantial economic costs. Examples of recent collapse of tunnels are the disaster in the Freeport mine where 33 workers got trapped 2013 [4], and the San José mine collapse when 33 men got trapped for more than two months [5]. In order to reduce the risk of tunnel collapses, various techniques are used to minimize instabilities in the tunnels.

One of the most commonly used techniques for stabilizing tunnels is the use of rock bolts. In the US alone, more than 100 million rock bolts are installed every year, as reported by Spearing et al. [6]. A rock bolt is a long metal rod which increases stability by transferring load from the outer layer (which has been damaged by mining activities such as blasting and drilling) to untouched rock several meters into the wall. To further increase the stability, specialized machines are used to spray shotcrete, a kind of liquid cement, on tunnel walls and ceilings. The shotcrete helps stabilizing the outer walls of a tunnel, and can be used either as-is or with steel fiberreinforcements. The fibers are usually small strips of metal which helps preventing cracks in the shotcrete. Metal wire nets can also be used to reinforce the shotcrete even further.

One problem is that rock bolts can become damaged by seismic activities or movements within the rock, and thus lose their loading capacity. If that happens, the outer shell of a tunnel's walls or ceiling can collapse, with disaster as a result. Therefore, there is a clear need of monitoring solutions for strain and thereby stress, as well as seismic and micro-seismic activity. By being able to localize areas where rock instability is increasing, and thus potential dangerous events can occur would dramatically enhance the working environment for workers, and reduce costs. For example, the use of strain measurements on rock bolts have previously been investigated by Schroeck et al. [7]. 
However, since the technique proposed in [7] is based on an offline approach where humans conduct the measurements, there is no possibility for continuous rock bolt monitoring. What is needed is a low-cost monitoring solution, capable of online monitoring rock bolts in near real-time.

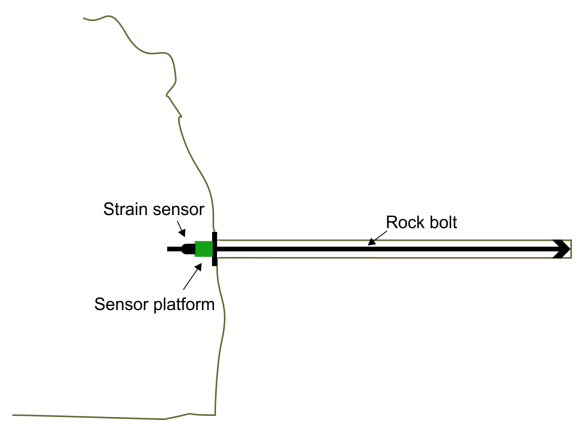

Fig. 1. Intelligent rockbolt in mine tunnel

In this paper, a novel method for mine monitoring is presented. The method is based on equipping standard rock bolts with Internet of Things technology, i.e. embedded electronics with the rock bolt as shown in Fig. 1. This approach enables real-time monitoring of each individual rock bolt in near real time. The current two monitored parameters are strain and seismic activities. Since no human involvement is required, and the intelligent rock bolts perform their operation without human involvement, the proposed approach can be deployed at a low cost compared to other solutions. The use of wireless IPv6 based communication and a Service Oriented Architecture (SOA) enable the rock bolts to be easily integrated in existing infrastructures and services, as shown in previous work [8]. The use of IP-based communication however introduces a security threat from hackers, viruses, malware, etc. Therefore is the Intelligent rock bolt supporting several communication security mechanisms for preventing malicious users and other threats from interfering with its operation.

This paper is outlined as follows: Section II presents a background and related work to the research. Section III outlines the proposed architecture followed by Section IV which presents the mine test setup. Results from the performed tests and plans for the next research steps are presented in Sections V and VI, respectively. Finally, the conclusions are presented in Section VII.

\section{BACKGROUND AND RELATED WORK}

Mine activity monitoring is today mostly made with geophones, still the most sensitive devices to detect earth movement [9]. In mines geo-phones are now interconnected and used to gather micro seismic data which is further analyzed to provide safety predictions [10], [11].

The mining industry have over time initiated a number of smaller projects to test the function of rock bolts. This has lead to some functional rock-bolt monitoring specifications [12], [13]. Some of the most important are:

- measure static and dynamic rock bolt load of $<300 \mathrm{kN}$.
- Dynamics to be captured are $<100 \mathrm{~Hz}$, thus a sampling rate of $1 \mathrm{kHz}$ will be sufficient.

- true load measured with an accuracy $2 \%$.

- not sensitive to uneven loading on the bolt plate.

- a cable free system.

- continuous load sampling over time (with the possibility to set sampling intervals).

- life time without changing power supply $>12$ month (using a battery).

There are several approaches to make one shot testing of rock bolts. Ultrasound is one common approach to measured bolt load through speed of sound measurements. We do find several scientific papers and several patents in this field. One example is [14]. Some suppliers of ultrasound measurement technology for bolt load measurements are:

- USM-3 by Norbar [15]

- Hevii - US bolt load technology [16].

- Boltscope-II by Hydratight [17]

This ultrasound technology has the potential to provide the most information on the changes in the rock bolt. The technology is still rather young and much development can be expected in the future. The major drawback is the price tag. An attractive approach for strain gauges sensing applied to rock bolt load measurements is the MMT prototype found with Hitec corporation [18]. They exhibit and custom device drilled into to the head of the bolt. The major draw back is the sensitivity to non-axial loads. To our understanding the development has been halted.

The process automation industry, where the use of sensors, actuators, distributed control systems and other technologies are widely used, have responded well to the new possibilities that networked embedded devices, e.g. Internet of Things (IoT) and Cyber-physical systems, (CPS) can offer [19]. The use of IP-based networked sensor and actuator devices with vertical integration into traditional industry systems is currently being investigated in some of Europe's largest automation projects such as the R\&D projects FP7 IMC-AESOP [20] and Artemis Arrowhead [21].

The COBS project [22] at Luleå University of Technology aims at developing smart conveyor belt rollers for the mining industry and logistics. By equipping a conveyor belt roller with a wireless sensor node and additional sensors, the roller is able to monitor itself and thereby sending alarms when for example a ball bearing is getting too warm which is an indication of a ball bearing damage. The higher level system is used to alert operators of any anomalies or alarms and assist in scheduling maintenance and reduces cost from less unexpected downtime.

\section{ARCHITECTURE}

This section outlines the core architecture of the intelligent rock bolt with its sensing and networking capabilities, the support for communication and as well as security.

\section{A. Intelligent rock bolt}

The current proposed design of the intelligent rock bolt is composed of several individual components. The base is 
a standard rock bolt, which is equipped with measurement electronics. The core of the electronic system is the Mulle platform from Eistec $\mathrm{AB}$ [23]. The Mulle is a low-power sensor node designed for Internet of Things applications. The current Mulle features a 16-bit microcontroller, analog and digital inputs and outputs, an 868 MHz IEEE 802.15.4 transceiver, several memories and power management circuits. To the Mulle is an interface board for the strain and vibration sensors connected, which is described in more detail in the next section. The Mulle runs the Contiki operating system from Dunkels et al. [24].

\section{B. Electronics and sensors}

The measurement system consists of a strain sensor and an accelerometer. The accelerometer is mounted on a printed circuit board (PCB) while the strain sensor is external to the $\mathrm{PCB}$, i.e. mounted inside the rock bolt's head. Both these sensors produce a voltage which is sampled by two 24-bit analog-to-digital converters (ADCs). These ADCs are mounted on the PCB which also hosts a connector that allows the ADCs to communicate with the Mulle using a high-speed SPI port.

The measurement board hosts a high-density connector for interfacing the Mulle. It also features some LEDs for development use, power supply, etc. Figure 2 shows the circuit of the vibration sensor system with Mulle platform.

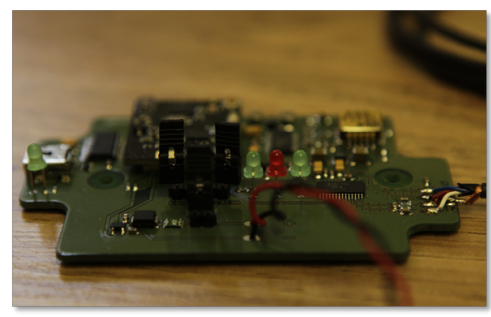

Fig. 2. Sensor node electronics

The two sensors, accelerometer and strain, have been chosen in order for the rock bolt to be able the two most important factors for mine stability. Seismic activity will cause vibrations in the rock, and forces lead to tensions in the rock which when released can result in small earthquakes. These quakes can in worst case result in the collapse of tunnels, or even portions of the mine.

\section{Internet of Things networking stack}

The current communication stack is based on previous work from several research projects. Other research projects that have been developing the Mulle architecture are EU FP7 IMC-AESOP and I2Mine and Artemis Arrowhead. The current version of the Mulle's communication stack is based on the IEEE 802.15.4 standard, and uses IPv6 and RPL over 6LoWPAN. Data is normally transmitted using SenML encoded using XML (with optional EXI-compression by the EXIP parser [25]) over CoAP. Figure 3 shows the Mulle's communication stack.
The software side of the strain and acceleration measurements were implemented as CoAP [26] services. A CoAP service is easily accessible through a web browser that supports it. This provides simplicity in monitoring and configuring the rock bolts as it can be done through a standard web interface over the Internet. CoAP is a protocol designed to be used on resource-constrained, low power electronic devices.

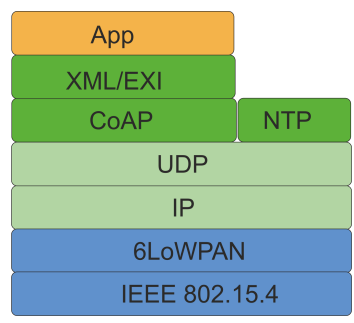

Fig. 3. Rock bolt communication stack

Since Contiki, which is used on the Mulle platform and hence rock bolts, runs RPL [27] it is possible to create mesh networks, i.e. with multi-hop support. The mesh networking support has been experimentally verified on the rock bolts and the performance of RPL has been investigated by Potsch et al. in [28]. Time synchronization is performed using the NTP protocol. Wireless re-programming of Mulle devices is handled by a custom written CoAP service. The Mulles are connected to existing networks (i.e. Ethernet) using a BeagleBone based gateway, also equipped with an IEEE 802.15.4 radio transceiver. The gateway host several services, such as RPL, NTP, and a number of CoAP services. The gateway is connected to its back-end system using an encrypted VPN solution. This ensures that sensor data is transmitted from a Mulle to database servers over encrypted channels only.

The use of a Ultra-wide Band (UWB) chip from Decawave has also been investigated. Preliminary results indicates that UWB is a viable solution for environments with severe multipath problems. This will be studied further as well. The use of UWB in combination with distributed event detection and pattern recognition, as proposed in [29], could provide one solution for performing detection and classification of mining related activities.

\section{Measurement software}

For the strain measurements, a CoAP service was created that can retrieve a strain sample at any time. Also, a threshold value can be set that allows the user of the service to be notified when a measurement is collected that has changed a specified amount from when the threshold was set. This is realized through CoAP's Observe-mechanism. Moreover, the sampling interval of the notifying service can be set through another CoAP service.

For the acceleration measurements, a CoAP service was created that controls the sensor to store a given amount of acceleration samples to the internal flash memory of the Mulle. When the logging is complete, the samples can be fetched through another service. Acceleration measurements are done 


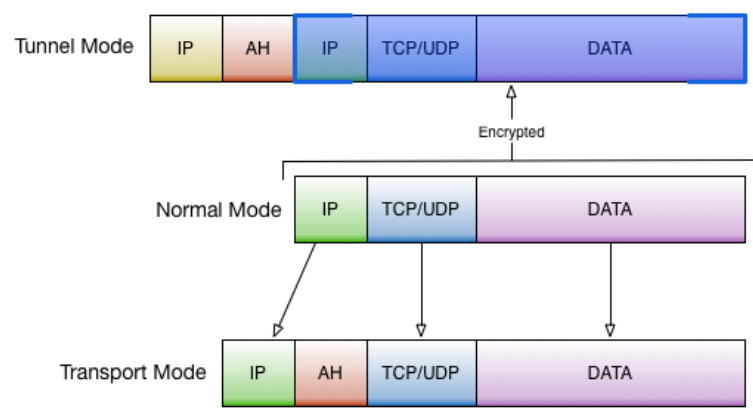

Fig. 4. IPsec encryption and authentication

in this way as acceleration data must be sampled at a much higher data rate than the available bandwidth of the wireless network.

\section{E. Communication security}

A high level of security usually means complex methods and algorithms, therefore more CPU time and more energy consumption. For this reason on low power systems (networks) the security design is a critical task. Nowadays one of the most extended systems over 6LoWPAN is IPsec that is an extension of the IP protocol that adds security to IP and higher layers. It was developed for the "new" IPv6 standard and was later adopted to include IPv4 as well.

IPsec has two different protocols, AH and ESP, to secure the authentication, integrity and confidentiality on communication [30]. IPsec can protect completely the IP datagram (Tunneling Mode [31]) or only the protocols on higher layers (Transport Mode). In Tunneling mode the IP datagram is encapsulated completely inside a new IP datagram that uses IPsec (the final IP of the datagram could even be different). In Transport mode, IPsec only manages the content of the IP datagram, adding the IPsec header between the original IP header and the header of higher layers, shown in Figure 4.

To protect the integrity of IP datagrams, the IPsec protocol uses authentication message codes based on hash, HMAC (Hash Message Authentication Codes). To protect the confidentiality of IP datagrams, IPsec uses standard algorithms of symmetric cipher (in our case using AES-128, but could work with any other cipher such as AES-256). In order to protect against DoS (Denial of Service) attacks, IPsec uses sliding windows. Each packet receives a sequence number and only is accepted by the receiver if the number of packet is inside this window or next. Any previous packets are immediately discarded. This is an efficient protection mechanism against attacks with message repetition, especially when the attacker is using sniffed original packets to resend.

The current IPsec version, which is based based on the compressed IPsec design developed by Raza [32], is under development and does not support directional keys, this means that IPsec must use a different secret key for each direction of the communication with the same client/server, but this implementation uses the same (reducing the security level).
One big step forward is the implementation of IKE - Internet Key Exchange - that is now work in progress. With IKE IPsec could change and choose the correct secret key for each communication. The use of DTLS encryption for CoAP would further increase the communication security [33].

\section{PERFORMED EXPERIMENTS}

This section presents the tests and experiments that have been performed, and gives an overview of all tests' setup in terms of hardware and software.

\section{A. Test overview}

In order to investigate the performance and feasibility of the rock bolt design, several tests were performed. The first set of tests was performed indoors in a controlled laboratory environment. When it was confirmed that the sensing electronics were functioning as planned as well the integration between the electronics and the rock bolt the next step was taken by performing tests using four rock bolts in an active mine. The mine test system was comprised of a total of four intelligent rock bolts, two Linux-based BeagleBone devices, interface cables, and power supplies.

\section{B. Laboratory test setup}

In the initial laboratory test, the strain sensor was mounted on a device constructed to simulate strain. This device was fastened to a desk and different torques were applied at the nut of the device to simulate the strain of a rock bolt. The accelerometer was also tested in a lab. setup where vibrations were measured as well. All measurement data were transmitted wirelessly using a CoAP service over a 6LoWPAN network and stored to file for later processing and visualization. A Java implementation of CoAP, Californium [34], was used to retrieve all measurements.

\section{Mine installation}

Figure 5 shows how an intelligent rock bolt is installed in a mine tunnel. The rock bolt itself is around three meters long, and the head with the Mulle and sensor interface board inside the grey plastic box. The strain sensor is located inside the stainless steel head. The two cables, one for power and one for data, are connected to the data logger and power supply, respectively. This installation is a prototype device, and not of production quality. In practice, the electronics must be protected in a better manner in order to withstand the harsh environment inside an active mine but for prototyping and testing this approach was sufficient.

\section{Performed tests}

When all four rock bolts were installed and equipped with the electronics for measuring strain and vibration, the two BeagleBone-based data loggers were time synchronized using NTP over an $100 \mathrm{Mbit} / \mathrm{s}$ Ethernet cable. Each logger stored data from one pair of rock bolts installed on the same tunnel wall. This procedure was performed during a total of three days in order to collect as much data as possible. 


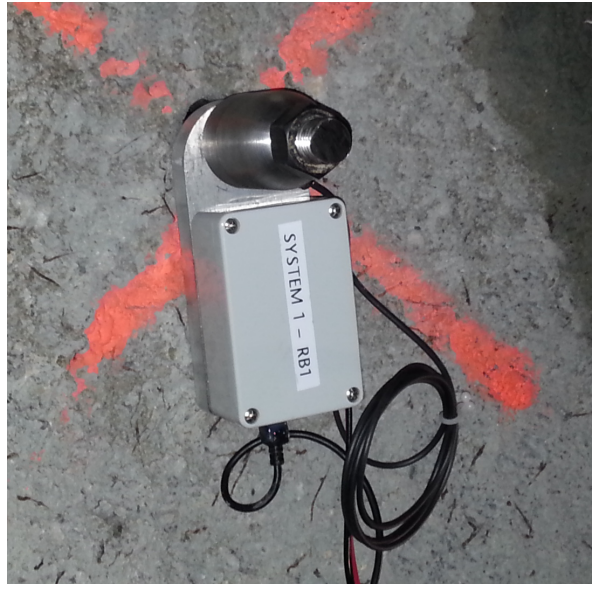

Fig. 5. Intelligent rock bolt installed in mine

Several different experiments were conducted in the mine in order to collect as much relevant data as possible. The performed experiments were:

1) Strain: The strain was recorded on all four rock bolts.

2) Tunnel wall vibration: A metal object was used as a hammer to hit the tunnel wall and the vibrations were recorded.

3) Top hammer drill rig: The vibrations generated by a production top hammer drill rig some 30 meters away for the rock bolts were recorded.

4) Falling rocks: A rock was dropped in order to simulate the event of rocks falling from a tunnel's ceiling.

5) Vehicle detection: A car was driven by the rock bolts and the generated vibrations were recorded.

\section{RESULTS}

This section presents results from the collected data from the laboratory experiments performed in August 2013 as well as from the Kittilä mine experiments performed in October 2013. All data processing and plots were performed using Matlab. For the accelerometer, the Z-axis has been used which corresponds to vibrations along the length of the rock bolt.

Note that a 24-bit ADC has been used, together with an accelerometer that can measure static acceleration (i.e. the gravity components is visible in the signal). A DC-blocking filter could be used to remove all offset. The accelerometer will see a different offset depending on the angle the rock bolt is installed with.

\section{A. Laboratory strain measurements}

To test the linearity of the strain sensor, different torques were applied to the strain simulation device. Four different boards and sensors were tested, labeled 2, 3, 4 and 5 and the strain output as a function of applied torque were recorded. The measurements were taken at torques of $0,40,50,70$ and $80 \mathrm{Nm}$. 10 measurements were taken for each value of torque and the mean and standard deviation, respectively, of the measurements were then plotted. The resulting plots are shown in Figure 6. It can also be seen that the strain measurement sensors have good linearity properties.

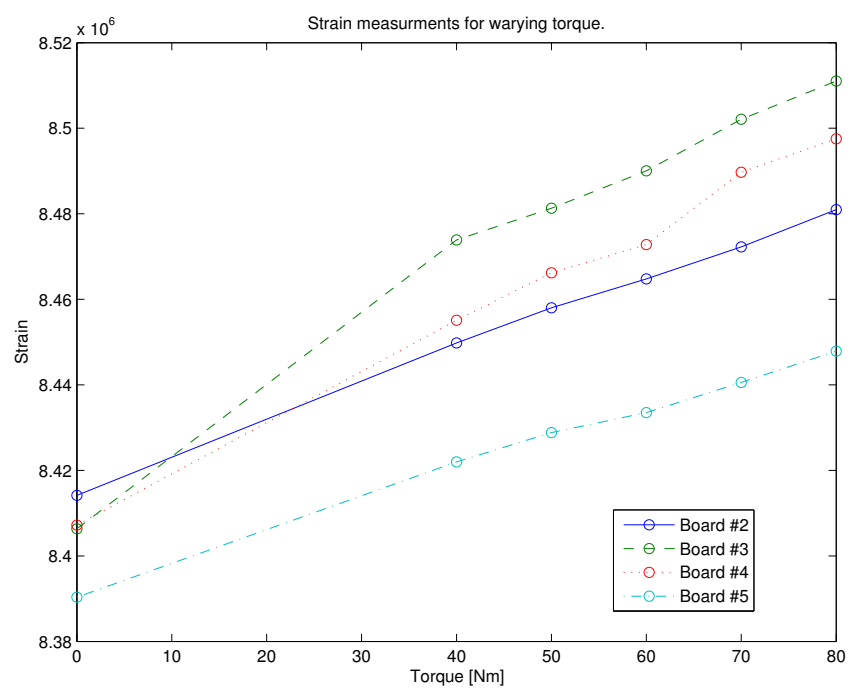

Fig. 6. Strain measurements for varying torque

\section{B. Steel rod}

In this first mine-based test, a rock bolt rod was used as a hammer to hit the wall near one of the installed rock bolts. This was repeated eight times in order to get a better understanding of which type of signal amplitudes that could be expected from a very strong source of vibration in close proximity of a rock bolt. The vibration data collected is shown in Fig. 7 .

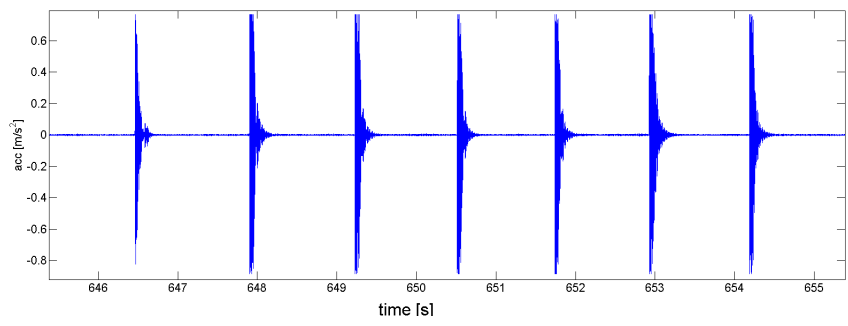

Fig. 7. Steel rod hit on wall

It is clearly shown in the wave form when the rod hits the tunnel wall and generates a vibration pattern. This type of amplitudes, or even higher, would probably also be generated if a mobile machine would drive too close to a wall and brush against it. The rock bolts can therefore be used for anomaly detection around vehicles.

\section{Drill test}

The second mine-based test was performed in order to investigate if a rock bolt's vibration sensor can be used to detect mining-related activities such as drilling. A mobile top hammer production drill rig, located approximately $25-30$ meters from the installed rock bolts, was used as a vibration source. 


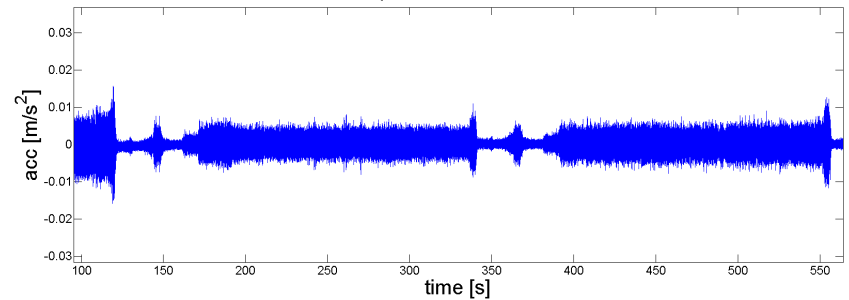

Fig. 8. Drilling detection

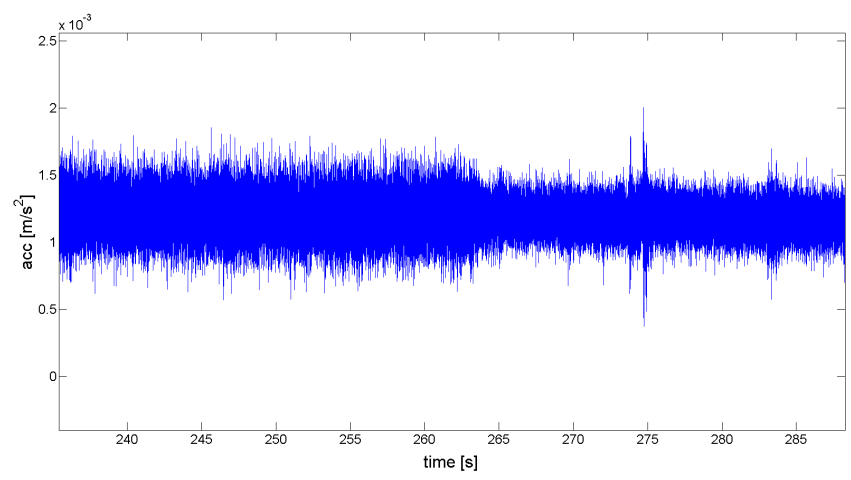

Fig. 9. Vehicle detection

It is clearly seen in the signal at 140 and 360 seconds in Figure 8 when the drilling machine drills, takes a short pause to insert a new rock tool, and starts drilling again. This indicates that a rock bolt can be used to detect drilling activity in close proximity, and even count how many drill holes that have been drilled.

\section{Vehicle detection}

One important feature that can be used to localizing vehicles is the ability for a rock bolt to monitor the presence of close by vehicles. This can be used for fine grain localization of mobile machinery such as cars, trucks, etc. Figure 9 shows the raw and unfiltered vibration signal from one rock bolt when a car was used in the vicinity. At 265 seconds into the signal, the car's engine was turned off which is clearly visible as a sharp drop of signal amplitude. The plotted signal is the raw output from the sensor, without any applied signal processing, such as filtering. By applying filtering techniques, the presence of a nearby vehicle could be detected [35].

How larger vehicles, such as loaders and trucks, will be observed is currently unknown. However, previous work performed within the iRoad project indicates that heavier vehicles generate higher amplitude levels, as shown by Hostettler et al. [36].

\section{E. Falling rock detection}

Rocks falling from a tunnel's ceiling are a clear indication of pending danger. When this occurs, a collapse of the tunnel could happen, or lead to larger and heavier rocks falling which could result in damage to vehicles and machinery as well as injuries on workers.

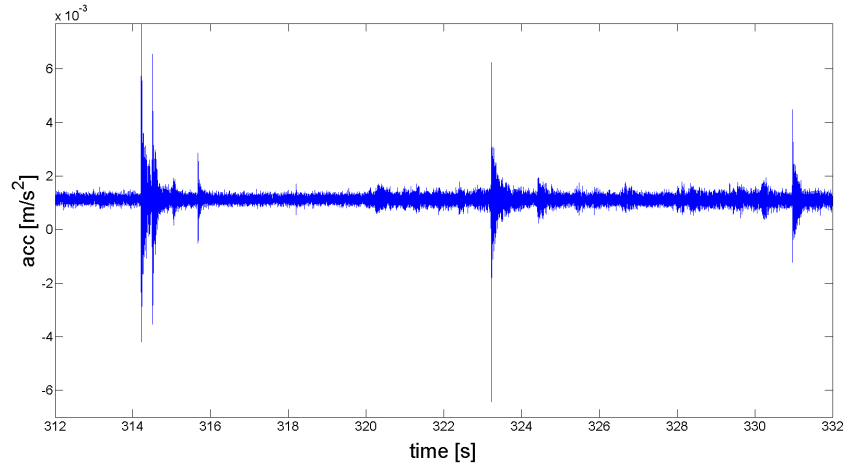

Fig. 10. Falling rocks detection

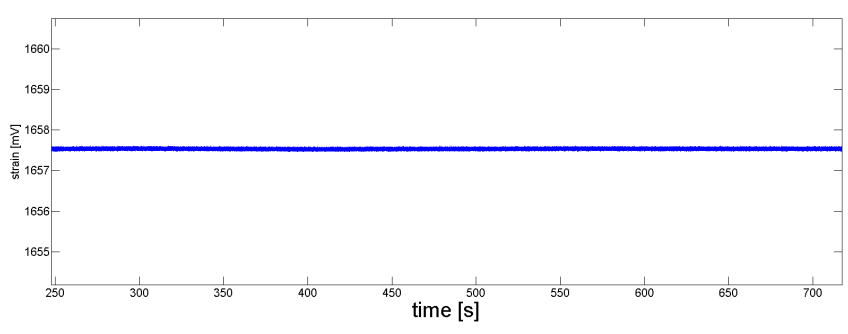

Fig. 11. Strain gauge sensor output

In order to see if the rock bolts could detect falling rocks, a simple experiment was performed by dropping a loose rock weighing approximately $3-4 \mathrm{~kg}$ from around two meters height down on the tunnel's floor around 1.5 meters from the rock bolt. At 314, 323 and 332 seconds in the signal shown in Fig. 10, three spikes are clearly visible. This indicates that an intelligent rock bolt can be used to detect falling rocks. When this feature is combined with the wireless communication capabilities, this could be used for a near real-time alarm system.

\section{F. Strain test}

A strain gauge sensor can be used to monitor stress in pillars, tunnels walls and ceilings. Strain can be a good indication of how strong forces that are affecting a volume of rock. The strain sensor is currently mounted at the rockbolt's head, however this will severely limit the amount of strain that can be detected by the sensor due to the fact that the shotcrete will limit the forces to propagate along the rockbolt.

The output from the strain sensor, shown in Figure 11, also concludes this. For better strain gauge sensor performance, the strain sensor must be re-designed. This is considered as future work.

\section{FUTURE WORK}

Some of the more prominent features that need more work are: efficient signal processing of captured data, sufficient low-power operation on sensing, processing and communication, and integration with back-end mine monitoring systems. Performance of the used sensors also needs more testing, especially the strain gauge which is challenging to observe in 
a mine due to the very high time constants and slow change rates. The mounting of the strain gauge also needs more investigation. Another key issue that needs more research is how the use of traditional Internet of Things protocols and technologies, which were originally designed for very low data-rate transmission, with no or low real-time requirement will behave when larger amounts of data must be streamed, i.e. from vibration sensors, with high requirements on low-latency transmission. The impact of scalability and security issues must also be investigated further. An interesting approach for self-learning methods for signal processing proposed in [37] would be interesting to evaluate for rock bolt usage. The fourth issue to explore is how strain and/or stress information and vibration data can be successfully integrated in today's monitoring systems.

In order to secure the communication and SOA model, the IPsec protocol must be enhanced with a key exchange mechanism like IKEv2 [38]. A system for fine-grain access control like Radius is also needed to be able to allow or deny specific clients to access services.

\section{CONCLUSION}

The use of rock bolts in the mining industry is a well known approach for increasing stability in for example tunnels, and thereby increasing safety for workers. However, what has been missing is a method of keeping installed rock bolts under constant monitoring. When compared to the process automation industry, where the use of sensors and SCADA system is a commonly used, rock bolt monitoring has not been especially improved.

This paper has presented a novel method for rock bolt monitoring, and the design of an intelligent rock bolt architecture with on-board sensing, processing and communication capabilities. The intelligent rock bolt, which comprise of a standard rock bolt, sensors and actuators, signal processing, data storage and wireless communication, can monitor itself and send alarms when seismic activities are detected, or when different mining activities are observed. Since security is highly important, the rock bolts have also been equipped with a security framework designed to provide tamper-free and secure communication. The rock bolt can detect, at least but not limited to, the following mining related activities:

- Deviation of strain on rock bolts

- Drilling

- Usage of mining machinery

- Falling rocks

This paper has also presented concrete test results from a mine-based field test using a low-cost intelligent rock bolt as the measurement device. Results from the tests indicates that a traditional rock bolt can be equipped with sensors, and that the sensors are capable of detecting mining-related activities.

Test results also show that successful integration between low-power electronics and a standard rock bolt is feasible. When all results presented in this paper are summarized, it is clear that intelligent rock bolts can be used within the mining industry to produce a better and safer working environment.

\section{ACKNOWLEDGMENT}

The authors would like to express their gratitude toward Agnico Eagle's Kittilä mine for allowing us to perform our experiments there. We would especially like to thank Antti Pyy and André van Wageningen for their assistance with the preparations and support during the field tests. The authors would also like to thank Mikael Larsmark for his contributions to the development of hardware and software for the intelligent rock bolt. The authors would also like to thank Fredrik Sandin for fruitful discussions regarding signal processing and data analysis.

We would also like to express our gratitude towards our partners within the I2Mine and Arrowhead projects, and the European commission and Artemis for funding. We would also like to thank Gluetech $\mathrm{AB}$ for assisting us with the strain gauge sensors and Eistec $\mathrm{AB}$ for their support with the Mulle platform.

\section{REFERENCES}

[1] "Mining and quarrying statistics - nace rev. 2,," Eurostat, Tech. Rep., 2013. [Online]. Available: http://epp.eurostat.ec.europa.eu/statistics_ explained/index.php/Mining_and_quarrying_statistics_-_NACE_Rev._2

[2] "The role of mining in national economies," ICCM - International council on mining and metals, Tech. Rep., Oct 2012. [Online]. Available: http://www.icmm.com/document/4440

[3] "Trends in the mining and metals industry," ICCM - International council on mining and metals, Tech. Rep., Oct 2012. [Online]. Available: http://www.icmm.com/document/4441

[4] "Indonesia freeport mine collapse," May 2013. [Online]. Available: http://www.reuters.com/article/2013/05/14/ us-indonesia-freeport-tunnel-idUSBRE94D06220130514

[5] "San jose mine collapse," August 2010. [Online]. Available: http: //www.theguardian.com/world/2010/aug/23/miners-trapped-alive-chile

[6] A. Spearing, B. Greer, and M. Reilly, "Improving rockbolt installations in US coal mines," Journal of the Southern African Institute of Mining and Metallurgy, vol. 111, pp. 555 - 563, 08 2011. [Online]. Available: http://www.scielo.org.za/scielo.php?script= sci_arttext\&pid=S0038-223X2011000800005\&nrm=iso

[7] M. Schroeck, W. Ecke, and A. Graupner, "Strain monitoring in steel rock bolts using fbg sensor arrays," pp. 298-304, 2000. [Online]. Available: http://dx.doi.org/10.1117/12.397895

[8] J. Eliasson, J. Delsing, A. Raayatinezhad, and R. Kyusakov, "A soabased framework for integration of intelligent rock bolts with internet of things," in Industrial Technology (ICIT), 2013 IEEE International Conference on, Feb 2013, pp. 1962-1967.

[9] C. E. Krohn, "Geophone ground coupling," Journal of GeoPhysics, vol. 49 , pp. $722-731,1984$.

[10] A. T. Kunnath and M. V. Ramesh, "Integrating geophone network to real-time wireless sensor network system for landslide detection," in Proc. First International Conference on Sensor Device Technologies and Applications, IEEE. IEEE, 2010, pp. 167-171.

[11] B. L. F. Daku and J. Salt, "Directional performance of an algorithm used to locate microseismic events in underground mines," in IECON 2011 - 37th Annual Conference on IEEE Industrial Electronics Society, Nov 2011, pp. 2198-2201.

[12] G. Bäckblom, "Project plan migs wp3 monitoring of rock bolt load in underground openings," RTC, Tech. Rep., Dec. 2008.

[13] J. Delsing, "Migs wp3 monitoring of bolt load - review of sensor technology for bolt load measurements," EISLAB, Luleå University of Technology,, SE-971 87 Luleå, Sweden, Tech. Rep., 2009.

[14] O. G. et.al., "Us patent 4,402,222, bolt load determining apparatus," Tech. Rep., Sept. 1983.

[15] [Online]. Available: http://www.norbar.com/

[16] [Online]. Available: http://www.heviitech.com/Hevii_UT.html

[17] [Online]. Available: http://www.hydratight.com/en/products/ultrasonics/ boltscope-ii 
[18] [Online]. Available: http://www.globalspec.com/Supplier/ CustomProductDetail/HITEC?Comp $=10 \& \mathrm{QID}=13910091 \&$ ExhibitId= 42329

[19] S. Karnouskos, O. Baecker, L. de Souza, and P. Spiess, "Integration of soa-ready networked embedded devices in enterprise systems via a cross-layered web service infrastructure," in Emerging Technologies and Factory Automation, 2007. ETFA. IEEE Conference on, Sept 2007, pp. 293-300.

[20] "IMC-AESOP - Architecture for Service-Oriented Process Monitoring and Control," Feb. 2013. [Online]. Available: http://www.imc-aesop.eu

[21] "Arrowhead - Enable collaborative automation by networked embedded devices.” Feb. 2013. [Online]. Available: http://www.arrowhead.eu/

[22] J. Eliasson, R. Kyusakov, and P.-E. Martinsson, "An Internet of Things approach for intelligent monitoring of conveyor belt rollers," in International Conference on Condition Monitoring and Machinery Failure Prevention Technologies - CM2013, June 2013

[23] "Eistec AB," Feb. 2013. [Online]. Available: http://www.eistec.se/

[24] A. Dunkels, B. Gronvall, and T. Voigt, "Contiki - a lightweight and flexible operating system for tiny networked sensors," in Local Computer Networks, 2004. 29th Annual IEEE International Conference on, Nov 2004, pp. 455-462.

[25] R. Kyusakov, H. Makitaavola, J. Delsing, and J. Eliasson, "Efficien $\mathrm{xml}$ interchange in factory automation systems," in IECON $2011-37$ th Annual Conference on IEEE Industrial Electronics Society, Nov 2011, pp. $4478-4483$.

[26] C. Bormann, A. Castellani, and Z. Shelby, "Coap: An application protocol for billions of tiny internet nodes," Internet Computing, IEEE, vol. 16, no. 2, pp. 62-67, March 2012.

[27] J. Tripathi, J. De Oliveira, and J. P. Vasseur, "A performance evaluation study of rpl: Routing protocol for low power and lossy networks," in Information Sciences and Systems (CISS), 2010 44th Annual Conference on, March 2010, pp. 1-6.

[28] T. Potsch, K. Kuladinithi, M. Becker, P. Trenkamp, and C. Goerg, "Performance evaluation of coap using rpl and lpl in tinyos," in New Technologies, Mobility and Security (NTMS), 2012 5th International Conference on, May 2012, pp. 1-5.

[29] M. Baqer and A. Khan, "Energy-efficient pattern recognition approach for wireless sensor networks," in Intelligent Sensors, Sensor Networks and Information, 2007. ISSNIP 2007. 3rd International Conference on, Dec 2007, pp. 509-514.

[30] V. Manral, "Cryptographic Algorithm Implementation Requirements for Encapsulating Security Payload (ESP) and Authentication Header (AH)," RFC 4835 (Proposed Standard), Internet Engineering Task Force, Apr. 2007. [Online]. Available: http://www.ietf.org/rfc/rfc4835.txt

[31] S. Kent, "IP Encapsulating Security Payload (ESP)," RFC 4303 (Proposed Standard), Internet Engineering Task Force, dec 2005. [Online]. Available: http://www.ietf.org/rfc/rfc4303.txt

[32] S. Raza, S. Duquennoy, T. Chung, D. Yazar, T. Voigt, and U. Roedig, "Securing communication in 6lowpan with compressed ipsec," in Distributed Computing in Sensor Systems and Workshops (DCOSS), 2011 International Conference on, June 2011, pp. 1-8.

[33] S. Raza, D. Trabalza, and T. Voigt, "6lowpan compressed dtls for coap," in Distributed Computing in Sensor Systems (DCOSS), 2012 IEEE 8th International Conference on, May 2012, pp. 287-289.

[34] M. Kovatsch, S. Mayer, and B. Ostermaier, "Moving application logic from the firmware to the cloud: Towards the thin server architecture for the internet of things," in Innovative Mobile and Internet Services in Ubiquitous Computing (IMIS), 2012 Sixth International Conference on, July 2012, pp. 751-756.

[35] R. Hostettler, W. Birk, and M. Nordenvaad, "Feasibility of road vibrations-based vehicle property sensing," Intelligent Transport Systems, IET, vol. 4, no. 4, pp. 356-364, December 2010.

[36] _ _ "Extended kalman filter for vehicle tracking using road surface vibration measurements," in Decision and Control (CDC), 2012 IEEE 51st Annual Conference on, Dec 2012, pp. 5643-5648.

[37] S. del Campo, K. Albertsson, J. Nilsson, J. Eliasson, and F. Sandin, "Fpga prototype of machine learning analog-to-feature converter for event-based succinct representation of signals," in Machine Learning for Signal Processing (MLSP), 2013 IEEE International Workshop on, Sept 2013, pp. 1-6. [Online]. Available: http: //pure.ltu.se/portal/files/43648751/mlsp2013.pdf

[38] C. Kaufman, P. Hoffman, Y. Nir, and P. Eronen, "Internet Key Exchange Protocol Version 2 (IKEv2)," RFC 5996 (Proposed Standard), Internet
Engineering Task Force, Sep. 2010, updated by RFCs 5998, 6989. [Online]. Available: http://www.ietf.org/rfc/rfc5996.txt

\section{BIOGRAPHY}

Dr. Jens Eliasson is an Associate Professor in Industrial electronics, and researcher in the field of Industrial Internet of Things and received his M.Sc. in Computer Engineering 2003, and Ph.D. in Industrial electronics 2008 at LuleåŨniversity of Technology in Sweden. He has since then been working with sensor networks and Internet of Things for industrial applications at the Embedded Internet Systems Laboratory (EISLAB). He is currently working with Service Oriented Architecture (SOA) for monitoring and control on deeply resource-constrained devices.

Pablo Puñal Pereira is a Ph.D. student in Mobile Internet of Things area at Luleå University of Technology in Sweden. He has received his 5 years degree in Physics in 2008 at the University of Santiago de Compostela, Spain. Also, he has a 5 years degree and a M.Sc. in Electronic Engineering obtained in 2011 at the University of Valencia, Spain. His main research areas involve security, authentication and services for low power wireless networks.

Prof. Delsing received the M.Sc. in Engineering Physics at Lund Institute of Technology, Sweden 1982. In 1988 he received the $\mathrm{PhD}$. degree in Electrical Measurement at the Lund University. During 1985 - 1988 he worked part time at AlfaLaval - SattControl with development of sensors and measurement technology. In 1994 he got the docent degree (associate prof) in Heat and Power Engineering. Early 1995 he was appointed full professor in Industrial Electronics at Luleå University of Technology where he currently is working as the scientic head of EISLAB, http://www.ltu.se/eislab. For the period 2004-2006 he also served as Dean of the engineering faculty at LuleåŨniversity of Technology. Since 1999 he is chairman of ITF (Instrument Tekniska Föreningen/Instrument Society of Sweden).

Joakim Nilsson is a Ph. D. student in Industrial Electronics at Luleå University of Technology in Sweden and received his M.Sc. in Engineering Physics and Electrical Engineering in 2013. He is currently doing research in the field of condition monitoring of power electronic devices at the Embedded Internet Systems Laboratory (EISLAB).

Joakim Gebart is working as a hardware design engineer at Eistec AB. Prior to this commitment, he was working as a software developer for safety-critical embedded systems in the defense industry. He has studied Applied Physics and Electrical Engineering at Linköping University and finished his M.Sc. thesis work in 2013. 